\title{
ВмJ Global Health A checklist to improve health system resilience to infectious disease outbreaks and natural hazards
}

\author{
Diane Meyer, ${ }^{1}$ David Bishai, ${ }^{2}$ Sanjana J Ravi, ${ }^{1}$ Harunor Rashid, ${ }^{3}$ \\ Shehrin Shaila Mahmood, ${ }^{3}$ Eric Toner, ${ }^{1}$ Jennifer B Nuzzo ${ }^{1}$
}

To cite: Meyer D, Bishai D, Ravi SJ, et al. A checklist to improve health system resilience to infectious disease outbreaks and natural hazards. BMJ Global Health 2020;5:e002429. doi:10.1136/ bmjgh-2020-002429

Handling editor Seye Abimbola

- Additional material is published online only. To view, please visit the journal online (http://dx.doi.org/10.1136/ bmjgh-2020-002429).

Received 28 February 2020 Revised 17 June 2020 Accepted 19 June 2020

\section{Check for updates}

(c) Author(s) (or their employer(s)) 2020. Re-use permitted under CC BY-NC. No commercial re-use. See rights and permissions. Published by BMJ.

${ }^{1}$ Environmental Health and Engineering, Johns Hopkins University Bloomberg School of Public Health, Baltimore, Maryland, USA

${ }^{2}$ Population, Family, and Reproductive Health, Johns Hopkins University Bloomberg School of Public Health, Baltimore, Maryland, USA

${ }^{3}$ Health Systems and Population Studies Division, ICDDRB, Dhaka, Bangladesh

Correspondence to

Diane Meyer;

dmeyer10@jhmi.edu

\section{ABSTRACT}

Recent infectious disease outbreaks, including the ongoing global COVID-19 pandemic and Ebola in the Democratic Republic of the Congo, have demonstrated the critical importance of resilient health systems in safeguarding global health security. Importantly, the human, economic and political tolls of these crises are being amplified by health systems' inabilities to respond quickly and effectively. Improving resilience within health systems can build on pre-existing strengths to enhance the readiness of health system actors to respond to crises, while also maintaining core functions. Using data gathered from a scoping literature review, interviews with key informants and from stakeholders who attended a workshop held in Dhaka, Bangladesh, we developed a Health System Resilience Checklist ('the checklist'). The aim of the checklist is to measure the specific capacities, capabilities and processes that health systems need in order to ensure resilience in the face of both infectious disease outbreaks and natural hazards. The checklist is intended to be adapted and used in a broad set of countries as a component of ongoing processes to ensure that health actors, institutions and populations can mount an effective response to infectious disease outbreaks and natural hazards while also maintaining core healthcare services. The checklist is an important first step in improving health system resilience to these threats, but additional research and resources will be necessary to further refine and prioritise the checklist items and to pilot the checklist with the frontline health facilities that would be using it. This will help ensure its feasibility and durability for the longterm within the health systems strengthening and health security fields.

\section{INTRODUCTION}

The ongoing COVID-19 pandemic has demonstrated the critical importance of resilient health systems in safeguarding global health security. In China, where the pandemic was first reported, for example, the health system was severely overwhelmed by the combination of a large surge of patients seeking care for respiratory symptoms and for other routine healthcare needs and reported lack of sufficient space, supplies and staff to

\section{key questions}

What is already known?

- Resilient health systems are critical to safeguarding global health security during public health emergencies.

- The human, economic and political tolls of public health emergencies can be amplified by health systems' inabilities to respond quickly and effectively.

- Frameworks, guidelines or other resources have not yet been developed that define the specific capacities and capabilities that health facilities and other actors involved in health services delivery should develop to prepare for significant infectious disease outbreaks and other public health emergencies.

What are the new findings?

- There are 10 thematic categories identified in our research as important components of resilient health systems, including core health system capabilities/ capacities, infrastructure/transportation, financing barriers to care, communication/collaboration/partnerships, leadership/command, surge capacity, risk communication, workforce and infection control.

- Achieving health system resilience within each of the 10 thematic categories requires numerous processes, mechanisms, policies and actions from a variety of different health system actors.

- Achieving health system resilience to outbreaks and natural hazards requires actions by facility and official-level actors.

\section{What do the new findings imply?}

- The health system resilience checklist can be used to help operationalise the concept of resilient health systems by allowing actors to measure the specific capacities, capabilities and processes that health systems need in order to ensure resilience in the face of both infectious disease outbreaks and natural hazards.

treat patients. Additionally, over 90000 health workers have reportedly been infected,${ }^{1}$ and one study found that nosocomial transmission was suspected in $41 \%$ of patients with confirmed severe acute respiratory syndrome 
coronavirus 2 infection. ${ }^{2}$ The dynamics of this ongoing outbreak exemplify the importance of strengthening health systems to improve outbreak preparedness, response and recovery, a sentiment that has been echoed by the WHO's Director General.

Unfortunately, this is not the first outbreak that has highlighted the role that vulnerable health systems can play in public health emergencies. Despite the ongoing outbreak of Ebola in the Democratic Republic of Congo occurring in a country with demonstrated expertise in controlling past Ebola outbreaks and widespread use of a candidate vaccine, to date approximately $5 \%$ of cases have occurred among healthcare workers ${ }^{3}$ and WHO has noted that healthcare facilities are serving as important drivers of disease transmission. Importantly, in both the Ebola and COVID-19 outbreaks, the human, economic and political tolls of these crises are being amplified by health systems' inabilities to respond quickly and effectively.

Improving resilience within health systems can build on pre-existing strengths to enhance the readiness of health system actors to respond to crises, while also maintaining core functions. However, the notion of resilience has been the subject of much debate and the literature on this subject remains largely at the conceptual level. For example, a recent review found that more work is needed to translate the concept of 'health systems resilience' into specific capacities and capabilities that health systems actors, such as health facilities, need to take to be ready for infectious disease outbreaks and other public health emergencies. ${ }^{4}$ Another found that lack of detail around the conceptualisation of health system resilience 'hinders the expansion of knowledge, the creation of reliable analytical tools and the effectiveness of communication' ${ }^{5}$ However, efforts to better define the concept, such as the work done by Abimbola and Topp, can help ensure a 'common understanding of the meaning of resilience' and help facilitate working towards common goals. ${ }^{6}$

Following the 2014-16 Ebola epidemic in West Africa, WHO developed a framework to help measure country progress towards reaching the International Health Regulations (IHR, 2005) target that each country has the capacity to prevent, detect and respond to public health emergencies. ${ }^{7}$ The WHO Joint External Evaluation (JEE) process and the results of these assessments have since been adopted by $>100$ countries and are now being used to gauge global readiness for significant infectious disease outbreaks and other public health emergencies. ${ }^{8}$ While the JEE assesses several public health capacities critical to health security, including laboratory biosafety practices, national laboratory systems and antimicrobial resistance, it does not assess many of the capacities and capabilities necessary for health system preparedness and response. ${ }^{4}$ Health systems play a direct and invaluable role in supporting countries' ability to respond quickly and efficiently to infectious disease outbreaks, including disease surveillance, detection and care of infected patients; they also play critical roles in mitigating the health impacts of other public health emergencies such as natural disasters and man-made catastrophes. Thus, it is critical that a health system's readiness for these events is also considered as countries strive to assess their overall preparedness. However, frameworks or tools that comprehensively identify the health system capacities and capabilities required for effective outbreak preparedness and response; define the roles and responsibilities of key health system stakeholders, such as health facilities and health service delivery organisations and articulate actionable steps for enhancing health system resilience have not yet been developed. ${ }^{4}$ As a result, countries that undergo a JEE without also taking steps to more explicitly evaluate their health systems do not actually have a comprehensive assessment of their readiness for potential public health emergencies.

Using data gathered from a scoping literature review, interviews with key informants and stakeholders who attended a workshop held in Dhaka, Bangladesh, we developed a Health System Resilience Checklist ("the checklist') to be used by health facilities, health services delivery organisations and national governmental health authorities. The aim of the checklist is to help operationalise the concept of health systems resilience by allowing health system actors to measure the specific capacities and processes needed in order to ensure resilience in the face of both infectious disease outbreaks and natural hazards. The checklist is intended to be adapted and used in a broad set of countries as a component of ongoing processes to ensure that health actors, institutions and populations can mount an effective response to infectious disease outbreaks and natural hazards while also maintaining core healthcare services. However, additional research will need to be conducted to further refine and validate the checklist. The checklist is modelled in part after prior work to define health system resilience within the US domestic context. ${ }^{910}$

\section{METHODS \\ Scoping review of the literature}

We conducted a scoping review ${ }^{4}$ to identify, through contributions within the peer-reviewed literature and on major organisational websites, what capacities (ie, systems, processes, policies, etc that are in place) and capabilities (ie, specific actions) are frequently associated with resilient health systems. We also sought to identify existing frameworks or checklists that measured these capacities and capabilities, and whether they overlapped with those identified in the WHO JEE tool.

Databases queried for the scoping review included PubMed, Web of Science and OAIster, and search terms were informed, in part, by the definition of a resilient health system developed by Kruk et al. ${ }^{11}$ Documents were included 'if they described health system capacities that could potentially strengthen health system to either infectious disease threats or natural hazards'. Documents were excluded if they only described public 
health capacities or if they addressed resilience within the context of other emergencies. All relevant articles were thematically coded, and, in total, 16 key themes were identified as "critical capacities necessary for health system resilience for infectious disease outbreaks and natural hazards'. ${ }^{4}$

\section{Key informant interviews}

Semi-structured interviews were conducted with key informants in the fields of healthcare preparedness, health systems strengthening, global health security, epidemic response and management and community and health sector resilience to gather their input on what health systems need to be resilient to infectious disease outbreaks and natural hazards. Interviewees were identified through the previously completed scoping literature review, existing professional relationships with individuals in related fields and previous work completed by the researchers.

An interview guide was developed prior to the start of the interviews to help direct the conversation. Each interview was conducted over the phone on a not-forattribution status and lasted approximately $45 \mathrm{~min}$. Interviews were taped with the interviewee's permission to help with thematic analysis. Themes were then iteratively discussed between research team members to identify those important to the development of the checklist. In total, interviews were conducted with 19 individuals who helped to shape and refine the initial draft checklist developed with information obtained from the scoping literature review.

\section{Checklist workshop-Dhaka, Bangladesh}

The pilot workshop took place in Dhaka, Bangladesh on 20-21 March 2019 and was held in partnership with icddr,b, an international health research organisation based in Dhaka. During the working group sessions, input and advice were solicited from stakeholders with detailed knowledge of resilience and health security to determine what capacities and capabilities are needed to ensure resilience during an infectious disease or natural hazard emergency within the context of Bangladesh's health system, government, population, infrastructure and geography.

A total of 65 participants attended the workshop inaugural address; 33 participants representing 16 different organisations attended the working group sessions over the course of 2 days. Stakeholders included representatives from icddr,b; the Institute of Epidemiology, Disease Control and Research; Save the Children; BRAC; Department of Disaster Management Studies; Dhaka University; SIDA; CARE; United States Centers for Disease Control and Prevention; Ministry of Health and Family Welfare and other government agencies with authority for response to emergencies and disasters, as well as governmental representatives involved in Bangladesh's JEE.
Checklist validation

During the validation exercise, 10 broad thematic categories, as well as associated capacities and capabilities, were presented to the attendees on index cards. These categories and associated capabilities/capacities (ie, 'checklist items') had been previously identified through the scoping literature review and key informant interviews. They were then asked to break into four small working groups (approximately six to seven individuals per group) and provide comments and suggestions on the proposed categories and checklist items, including whether they would omit or add any categories or checklist items. The checklist was then refined by the research team to include the attendees' edits and/or observations for the subsequent workshop session on checklist prioritisation.

\section{Checklist prioritisation}

The workshop attendees were then asked to prioritise the revised checklist items based on what capacities and capabilities they thought, given their broad expertise, should be of highest priority using a $1-5$ scale ( $5=$ highest priority, $1=$ lowest priority). Attendees were also asked to note with an asterisk which of the checklist item(s) they thought were of greatest importance (ie, If they could only choose to implement a couple of the checklist items, which would they choose?).

\section{Patient and public involvement}

Neither patients nor the general public were involved in the design, conduct, reporting or dissemination of our research.

\section{FINDINGS}

The final Health Systems Resilience Checklist is presented in online supplementary table 1 . The checklist is meant to identify those capacities and capabilities necessary for health system resilience to infectious disease outbreaks as well as natural hazards. We focus consideration on infectious disease outbreaks that can have significant impacts on the functioning of a health system, including highconsequence infectious diseases, which require complex medical management and special infection control considerations for specific medical procedures (eg, aerosol-creating procedures like placement of central lines and intubation). For example, this would include outbreaks of viral haemorrhagic fevers such as Ebola and Lassa.

The checklist is divided into 10 thematic categories which were informed and refined by the scoping literature review, ${ }^{4}$ key informant interviews and pilot workshop checklist validation exercise. No thematic categories were added or removed by participants during the validation exercise. Each thematic category is accompanied by a list of specific capacities and capabilities (ie, 'checklist items') that were identified as important to achieving resilience within the larger theme. In total, only three checklist items were removed by working group members, as those 
were not viewed to be important for health system resilience within the context of Bangladesh's health system. Forty checklist items were kept as originally written, and 53 were either modified or added.

The 10 thematic categories and their descriptions are:

1. Core health system capacities and capabilities: the capacities and capabilities needed to maintain core functioning during a public health emergency, such as access to maternal-child care.

2. Critical infrastructure and transportation: the infrastructure (eg, water, sanitation) and transportation that must be in place to ensure continued functioning during an emergency.

3. Financing: the presence of adequate resources to both maintain routine services and to respond to public health crisis.

4. Barriers to accessing health services: barriers that exist that might prevent individuals from accessing care routinely and during public health emergencies.

5. Communication, collaboration, coordination and partnerships: relationships that exist with response partners before and during a public health emergency.

6. Leadership and command structure: the leadership, command and incident management structures and policies needed to respond to a public health crisis.

7. Surge capacity: policies, practices and systems necessary to accommodate a surge of patients during a public health emergency.

8. Risk communication: policies and practices for communicating and engaging with the public about public health emergencies.

9. Workforce: the health workforce and associated capacities and capabilities, including training and support, needed to respond to a public health emergency.

10. Infection control: the infection control processes and procedures needed to prevent spread of disease and screen and treat patients within facilities.

The checklist items identified within each theme are divided up by the actor typically responsible for a given checklist item in most settings. For the purposes of this checklist, we identified two specific actor categories:

1. Facility-level actors: these include individual health facilities, such as clinics and hospitals, as well as larger health system networks and healthcare organisations.

2. Health official-level actors: these include provincial, regional or national-level health officials, such as ministries of health.

For some of the checklist items, there are actions necessary at both the facility and health official level. However, for others, there may not be a corresponding action for each actor.

The checklist items are phrased as statements such that users can use a Likert scale to indicate whether they believe a given capacity currently exists within their facility or institution (ie, $0=$ disagree, $1=$ somewhat agree, $2=$ strongly agree). The checklist also contains a column of suggested resources (see online supplementary appendix A for full citations of resources) that might help users better develop a given capacity or capability. The resources were identified through the data collection process and through prior knowledge of the research team. While many of the checklist items include the drafting or existence of plans, protocols or systems, it is also expected that these protocols or plans have been disseminated to the appropriate individuals who might need them during a public health emergency, and that all requisite personnel have been trained on how to use a given system. Finally, the checklist has been tiered according to how advanced a given capacity or capability is, based on the experience of the research team. The most feasible, fundamental core capacities/capabilities necessary for resilience are tiered as a 1 , followed by tiers 2 and 3, which identify more advanced capacities/capabilities necessary for resilience. The tiers are presented in parenthesis next to the associated checklist item, but additional research will be needed to refine these tiers with experts working in health system development.

During the second workshop session, participants were asked to prioritise each of the checklist items ( 5 =highest priority, $1=$ lowest priority). Those designated as a 4 or 5 meant that development of that checklist item(s) should be prioritised. Online supplementary table 2 presents the average and median score for each of the checklist items. Only eight of the checklist items had an average score of $<4$. The checklist items starred by two or more of the small working groups as highest priority for health system resilience are provided in table 1. Importantly, there was consistency among the four smaller working groups in both prioritisation scores and in which checklist items were starred as highest priority.

\section{DISCUSSION}

Resilient health systems are important for supporting response efforts during an infectious disease outbreak or natural hazard, and help ensure the continued delivery of routine services needed by the community in nonemergency periods. However, health systems cannot achieve resilience without first identifying what capacities and capabilities are necessary to respond to these threats. We have created a health systems resilience checklist as a critical first step in advancing health system preparedness beyond just descriptions of high-level attributes to define specific capacities and capabilities. We recognise that many of the checklist items will require significant investments, including time, money and personnel, and are an enormous undertaking for most institutions. However, we hope the checklist will help ignite the strategic planning processes necessary for improving health system resilience to infectious disease outbreaks and natural hazards for the long-term.

One of the goals of our work to develop the checklist was to determine the extent to which efforts to advance health security address foundational work in health systems strengthening. To advance health security, it will be essential that existing efforts to ready countries 
Table 1 Checklist items identified by two or more working groups as highest priority for health system resilience

\begin{tabular}{|c|c|}
\hline System for reporting & This facility currently has a system for reporting infectious disease cases \\
\hline Sufficient financing & $\begin{array}{l}\text { This facility currently has sufficient financing available to provide essential core health } \\
\text { system capacities. }\end{array}$ \\
\hline Protocols for hazardous waste & $\begin{array}{l}\text { This facility currently has protocols (and has disseminated these protocols) for handling of } \\
\text { infectious/hazardous waste. }\end{array}$ \\
\hline $\begin{array}{l}\text { Plans for distributing emergency } \\
\text { funding }\end{array}$ & $\begin{array}{l}\text { Health administrators currently have plans (and have disseminated these plans) for } \\
\text { authorising and distributing emergency funding to health facilities (eg, to acquire additional } \\
\text { supplies and resources, ensure healthcare worker compensation) both at the central and } \\
\text { local levels. }\end{array}$ \\
\hline $\begin{array}{l}\text { Established relationships with } \\
\text { community }\end{array}$ & $\begin{array}{l}\text { This facility has engaged and established relationships with community leaders and } \\
\text { decision makers to help foster community trust in the healthcare system. }\end{array}$ \\
\hline $\begin{array}{l}\text { Plans for coordinating with other } \\
\text { facilities }\end{array}$ & $\begin{array}{l}\text { This facility currently has plans (and has disseminated these plans) on how to coordinate } \\
\text { with other facilities (including public and private) in the event that it needs to transfer } \\
\text { patients or share supplies. This might be accomplished through the creation of } \\
\text { memorandums of understanding. }\end{array}$ \\
\hline Established leadership hierarchy & $\begin{array}{l}\text { This facility has established leadership within each level of the health system (ie, local, } \\
\text { district, regional). }\end{array}$ \\
\hline $\begin{array}{l}\text { Represented at Emergency } \\
\text { Operations Centre (EOC) }\end{array}$ & $\begin{array}{l}\text { The Ministry is represented at the EOC and is part of the official command structure, and } \\
\text { there is an organised network of healthcare centres that can feed into the EOC. }\end{array}$ \\
\hline Support workforce training & $\begin{array}{l}\text { Health administrators can currently support the quick healthcare workforce training } \\
\text { necessary for outbreak response, including proper infection control practices and how to } \\
\text { treat, isolate and report cases. }\end{array}$ \\
\hline
\end{tabular}

is linked with health system-strengthening efforts. This approach could help strengthen foundational health system capacities and capabilities that are also needed to support public health responses to crises. Similarly, efforts to strengthen health systems can be significantly undermined if they do not adequately plan for potential crises such as infectious disease outbreaks and natural hazards.

The WHO's JEE process has helped to motivate progress towards measurement of countries' capacity to prevent, detect and respond to public health threats. Since 2016, it has been implemented in over 100 countries, with many more planning on completing one in the next few years. Despite this important progress, a previous review determined that the JEE tool does not adequately address capacities and capabilities specific to health systems, leaving a gap in its overall ability to measure global health security. Consequently, countries that undergo a JEE and make improvements based on their JEE results could remain unprepared for public health emergencies if they have not also adequately assessed the readiness of health facilities and other components of the broader health system.

Although events like the ongoing COVID-19 and Ebola crises illustrate the interconnectedness between health systems strengthening and health security, there is little overlap between programmatic efforts to advance health security and to strengthen health systems. In addition to a lack of inclusion of health systems perspectives within the JEE tool, often health system strengthening experts are largely unaware of the JEE process and other ongoing efforts to advance global health security. This lack of mutual awareness results in missed opportunities to build on the achievements of both disciplines to advance health system resilience.

The key to advancing health systems resilience will be to assess the extent to which health systems have the capacities and capabilities needed to respond to emergencies while also maintaining other routine services, that, if neglected, could lead to increased morbidity and mortality. This checklist represents the beginning of a process to define the specific capacities and capabilities that health systems require to be resilient in the face of infectious disease outbreaks and natural hazards. In developing this checklist, we aimed to advance thinking about health systems resilience beyond basic definitions of high-level attributes of resilient systems to imagining processes for transforming public health practice at the administrative, governmental and health facility levels. Additional workshops will be needed, including with frontline healthcare providers, to further refine and validate the checklist. Conducting similar workshops in other countries will also be necessary to assess the checklist's adaptability across multiple country contexts.

Although the checklist includes some guidance for implementation, several of the recommended activities may still be quite challenging for health systems to execute and will likely require additional guidance and technical support, such as allocation of scarce resources during an emergency-an ethically fraught issue for health system stakeholders to address. As a practical matter, the feasibility of implementing each item will differ across various locations and contexts; even so, the 
checklist can still serve as a starting point for the development of more contextualised local action plans.

Finally, countries cannot build and maintain these capacities and capabilities without also addressing the structural, economical and political barriers that have hindered the development of resilient health systems across the globe. This includes, among many others, ensuring that all individuals have access to healthcare services ${ }^{12}$ and identifying and addressing social determinants of health. ${ }^{13}$ By addressing these fundamental gaps, countries can enhance the health system infrastructure that will be critical to operationalising this checklist and strengthening global health security.

\section{Future uses of a checklist}

We present above a literature-derived and key informant and practitioner-informed checklist for evaluating and monitoring health systems resilience for infectious disease outbreaks and other public health emergencies. However, more work is needed to transform this into a checklist that is used to assess and improve resilience. A checklist differs from a conceptual framework because it is meant to be applied in service of enhancing the capacity and coherence of workers throughout a system. As such, it must accompany a system for distribution and practical application. A checklist is intended to guide participatory self-assessment, flag gaps and motivate effort to close gaps. Thus, a checklist that is not owned, adapted, acted on or implemented by the intended end users will not transform weak health systems into resilient ones.

More work is needed to link this proposed checklist to efforts to strengthen health systems resilience. Improving the quality of professional practice is always challenging because it involves a complex mix of measurement, motivation, skills and relationships. Success in studying systems improvement requires a rigorously applied benchmark for the intended system goals and consistent attention to sharing and spreading the goals throughout the system.

It is important to ensure that checklists aimed at improving preparedness are tailored to the environments in which they are to be used and accepted by potential users. Previous studies have shown that the specific barriers and facilitators to system improvement vary across stakeholders and facilities. ${ }^{14}$ Hence, 'tailored' performance improvement interventions have had growing appeal. Tailored approaches require direct contact for evidence gathering prior to select specific remedies to specific performance priorities. ${ }^{14} \mathrm{~A}$ recent systematic review of tailored interventions for health worker performance faced daunting levels of heterogeneity in the types of tailored interventions that it sought to pool for analysis. The spectrum ranged from disseminating papers, delivering lectures, offering decision support tools like checklists as well as using audit and feedback via supportive supervision guided by a checklist. ${ }^{14}$ Tailoring was done in so many specific ways across the 32 trials included in the systematic review that no common conclusion on efficacy could be reached. The systematic review concluded that tailored approaches to professional practice improvement can be effective but effect sizes varied with the context and setting. ${ }^{14}$

Checklists are meant to improve preparedness; therefore, it is essential that they are coupled with a process for monitoring and evaluating their impacts. Audit and feedback (A\&F) is a specific method of supportive supervision that uses checklists. It tasks the supervisor with collecting systematic information about practice and discussing it with the professional in a manner to support positive change. The most recent systematic review found that A\&F leads to a median $4.3 \%$ absolute improvement in performance-large effects were found in $25 \%$ of studies and null effects were found in $25 \% .^{15}$

Thus, there is a continuum of future applications of the checklist from the least expensive, least transformative method of simply sharing the checklist within a system, to a more effective deployment in which supportive coaches are distributed throughout the system to build long-lasting relationships with staff. By assigning staff to conduct recurrent reviews of their own checklist performance one can design work plans to prioritise capacity building resources. Based on these findings, we note that further work must be conducted to assess the applicability and acceptability of our proposed checklist in other settings, and acknowledge the need to assess its impact in settings where it has been introduced as part of a comprehensive approach to improved preparedness. What is learnt during application of the approach should be used to iteratively update and refine the checklist as needed.

\section{CONCLUSIONS}

Resilient health systems are critical to enabling robust responses to outbreaks and natural hazards while also sustaining baseline functions. The development of this checklist is an important first step in improving health system resilience to such threats, but additional research and resources will be necessary to further refine and prioritise the checklist items, and to pilot the checklist with frontline health facilities. This will help ensure its feasibility and durability for the long-term within the health systems-strengthening and health security fields.

Acknowledgements The authors would like to thank Michael Snyder for his help with this project and the Rockefeller Foundation for funding this project. The Rockefeller Foundation promotes the resilience concept across different domains including urban planning, climate adaptation and the health sector.

Contributors JBN and DB conceived of and led this research. JBN, DB, DM and SJR conducted the scoping review and subject matter expert interviews. JBN, DB, DM, SSM and HR organised and led the working group meetings. JBN, DB, $\mathrm{DM}, \mathrm{SSM}$ and HR analysed the working group meeting data. DM drafted the initial manuscript, and JBN, DB, DM, SJR, SSM, HR and ET reviewed and revised the manuscript.

Funding This research was supported by the Rockefeller Foundation.

Competing interests None declared.

Patient consent for publication Not required.

Provenance and peer review Not commissioned; externally peer reviewed.

Data availability statement Data sharing not applicable as no datasets generated and/or analysed for this study. 
Open access This is an open access article distributed in accordance with the Creative Commons Attribution Non Commercial (CC BY-NC 4.0) license, which permits others to distribute, remix, adapt, build upon this work non-commercially, and license their derivative works on different terms, provided the original work is properly cited, appropriate credit is given, any changes made indicated, and the use is non-commercial. See: http://creativecommons.org/licenses/by-nc/4.0/.

\section{REFERENCES}

1 ICN. Icn calls for data on healthcare worker infection rates and deaths. Available: https://www.icn.ch/news/icn-calls-datahealthcare-worker-infection-rates-and-deaths [Accessed 8 May 2020].

2 Wang D, Hu B, Hu C, et al. Clinical characteristics of 138 hospitalized patients with 2019 novel coronavirus-infected pneumonia in Wuhan, China. JAMA 2020. doi:10.1001/ jama.2020.1585. [Epub ahead of print: 07 Feb 2020].

3 WHO. Coronavirus disease 2019 (COVID-19) situation Report-90, 2020. Available: https://www.who.int/emergencies/diseases/ebola/ drc-2019/situation-reports

4 Nuzzo JB, Meyer D, Snyder M, et al. What makes health systems resilient against infectious disease outbreaks and natural hazards? Results from a scoping review. BMC Public Health 2019;19:1310.

5 Turenne CP, Gautier L, Degroote S, et al. Conceptual analysis of health systems resilience: a scoping review. Soc Sci Med 2019;232:168-80.
6 Abimbola S, Topp SM. Adaptation with robustness: the case for clarity on the use of 'resilience' in health systems and global health. BMJ Glob Health 2018;3:e000758.

7 World Health Organization. Joint external evaluation tool. International health regulations (2005); 2016.

8 Global Preparedness Monitoring Board. A world at Risk-Annual report on global preparedness for health emergencies; 2019.

9 Toner ES, McGinty M, Schoch-Spana M, et al. A community checklist for health sector resilience informed by Hurricane sandy. Health Secur 2017:15:53-69.

10 Meyer D, Kirk Sell T, Schoch-Spana M, et al. Lessons from the domestic Ebola response: improving health care system resilience to high consequence infectious diseases. Am J Infect Control 2018;46:533-7.

11 Kruk ME, Myers M, Varpilah ST, et al. What is a resilient health system? lessons from Ebola. Lancet 2015;385:1910-2.

12 Ooms G, Beiersmann C, Flores W, et al. Synergies and tensions between universal health coverage and global health security: why we need a second 'Maximizing Positive Synergies' initiative. BMJ Glob Health 2017;2:e000217.

13 Labonté R. Health systems governance for health equity: critical reflections. Rev Salud Publica 2010;12 Suppl 1:62-76.

14 Baker R, Camosso-Stefinovic J, Gillies C, et al. Tailored interventions to address determinants of practice. Cochrane Database Syst Rev 2015:CD005470.

15 Ivers NM, Sales A, Colquhoun $\mathrm{H}$, et al. No more 'business as usual' with audit and feedback interventions: towards an agenda for a reinvigorated intervention. Implement Sci 2014;9:14. 